\title{
Business oder Life Engineering?
}

\author{
Hubert Oesterle
}

Eingegangen: 8. September 2014 / Angenommen: 28. September 2014 / Online publiziert: 5. November 2014 (C) Die Autor(en) 2014. Dieser Artikel ist auf Springerlink.com mit Open Access verfügbar

Zusammenfassung Die Digitalisierung der Konsumentenwelt bringt einen Paradigmenwechsel für Unternehmen: Erfolgreiche digitale Dienste entstehen aus Sicht der Lebensbereiche von Konsumenten; die Leistungserstellung im Unternehmen leitet sich daraus ab. Die Digitalisierung der Welt legt uns jedoch nahe, über einen zweiten Paradigmenwechsel nachzudenken, also nicht nur dem Paradigma der Konsumentenzentrierung aus Sicht des Geschäftserfolgs, sondern auch dem Paradigma der Lebensqualität nachzuleben. Die Konsequenz aus diesem Paradigmenwechsel wäre eine Disziplin des Life Engineering, nicht anstatt, sondern zusätzlich zum Business Engineering, das weiterhin für Effizienz in der Befriedigung der Bedürfnisse sorgen muss. Life Engineering beschäftigt sich mit der Lebensqualität, den Bedürfnissen und den Möglichkeiten zu deren Befriedigung sowie dem Beitrag der digitalen Services. Erste Ansätze des Life Engineerings werden am Beispiel eines digitalen Dorfes aus dem Forschungsprojekt „Independent Living“ veranschaulicht. Aus den bisherigen Erkenntnissen, lässt sich eine Umsetzungs- und Forschungsagenda ableiten. Ob die Wirtschaftsinformatik sie aufgreift, bleibt abzuwarten.

Schlüsselwörter Business Engineering · Life Engineering · Geschäftsmodelle · Digitale Services $\cdot$ Konsumentenzentrierung $\cdot$ Lebensqualität

\section{Digitale Services}

Google, Apple, Amazon, Facebook, Tencent und viele weitere, vorwiegend junge Unternehmen digitalisieren die Welt der Konsumenten. Klassische Organisationen

H. Oesterle $(\bowtie)$

Universität St. Gallen,

St. Gallen, Switzerland

E-Mail: hubert.oesterle@unisg.ch

를 Springer 
wie öffentliche Verwaltung, Banken und Medien tragen dazu durch Digitalisierung ihrer bestehenden Marktleistungen bei.

Die große Herausforderung für alle Beteiligten ist die Frage: Was davon nützt den Menschen? Die Antwort auf diese Frage geben nicht die technischen Möglichkeiten, sondern das reale Leben von Menschen wie im folgenden Beispiel.

Frau Macher (42 Jahre), Mutter einer zwölfjährigen Tochter und eines zehnjährigen Sohnes, nutzt einen Schlafmonitor auf dem Smartphone, der aufgrund von Bewegungsmustern im Schlaf einen Moment zum Wecken auswählt, der ihr den Einstieg in den Tag erleichtern soll. Im Badezimmer erinnert sie ein Pill Reminder an die Einnahme eines Medikaments. Beim Frühsport auf dem Crosstrainer ermittelt ein Sensor die Herzfrequenz bei unterschiedlichen Belastungen und gibt Hinweise zur Fitness. Vor dem Anziehen ruft Frau Macher die Wettervorhersage und die Außentemperatur sowie die Termine des Tages ab, um die richtige Kleidung auszuwählen.

In ihrer Teilzeitbeschäftigung als Pharmavertreterin richtet sich ihr Tagesablauf nach den Terminen mit Ärzten und Apotheken. Beim Frühstück erhält sie die Mitteilung, dass ein Arzt kurzfristig einen Termin abgesagt hat. Eine App zur Terminvereinbarung, die Zugriff auf ihren Kalender und die Kontaktdaten des Arztes hat, schlägt ihr drei neue Termine vor, von denen sie zwei von der App an den Arzt weiterleiten lässt.

Die Tochter klagt, dass ihre Lieblingsmarmelade ausgegangen ist. Frau Macher scannt mit ihrem Smartphone den Barcode des Glases und fügt das Produkt in die Einkaufsliste für Samstag ein.

Das Navigationssystem auf ihrem Smartphone hilft ihr bei der Routenwahl unter Berücksichtigung der voraussichtlichen Verkehrssituation. Ihr Auto warnt sie vor Überschreitungen des Tempolimits und dem Verlassen ihrer Fahrspur. Im Wartezimmer des Arztes informiert sie sich anhand des CRM-Systems ihres Unternehmens über den Arzt, seine Umsätze, seine Teilnahme an Weiterbildungsveranstaltungen, seine Hobbies (aus den sozialen Netzwerken), über seine Freunde, seine Möglichkeiten als Meinungsmacher usw.

Am Abend hilft sie ihrem Sohn bei einer Zinseszins-Hausaufgabe, nachdem sie sich selbst auf Wikipedia die Grundlagen nochmal angeschaut hat. Mit ihrer Tochter sucht sie im Internet nach Möglichkeiten zur Augenkorrektur durch Laser oder Einsetzen von Linsen sowie nach Erfahrungsberichten und Studien dazu. Anschließend erledigt sie mit ihrem Mann die Reservationen für ihren Skiurlaub zusammen mit Freunden.

Schließlich bezahlt sie zwei Rechnungen und beantwortet ein paar E-Mails, geschäftliche und private. In einem sozialen Netzwerk betrachtet sie noch Urlaubsfotos von Freunden. Zur Entspannung liest sie schließlich einen Roman auf dem E-Book-Reader.

Apps auf Smartphones und anderen mobilen Geräten, auf Spielkonsolen und auf Fernsehern, interaktive Websites und intelligente Geräte bieten uns Millionen von digitalen Services an. Sensoren für Temperatur, Ort, Bewegung usw. erfassen unzählige Daten in Echtzeit, viel genauer und detaillierter als je zuvor. Haussteuerungen, Autos, Roboter und andere Geräte setzen diese Daten teilweise autonom in Aktivitäten um. Wie weit wir diese Möglichkeiten zur Verbesserung unserer Lebensqualität nutzen, hängt von unserer Persönlichkeit, unserer Lebenssituation und natürlich 
von der Qualität der Services ab. Analysieren Menschen unserer hoch entwickelten Gesellschaften ihre Tagesabläufe, sind sie überrascht, wie viele digitale Dienste sie bereits nutzen.

Websites wie distimo (s. dazu IL.iwi.unisg.ch/AppMap, Productivity, App Selection) bieten einen Überblick über die Welt der digitalen Services, meist der mobilen Apps, allerdings aus Sicht der Anbieter. Wir haben eine konsumentenzentrierte Sichtweise eingenommen, aus dem Alltag der Menschen Lebensbereiche abgeleitet und diesen anschließend digitale Services zugeordnet. Abbildung 1 zeigt zehn Bereiche sowie für den Bereich Shopping eine mögliche Verfeinerung bis auf die Ebene einzelner Dienste, also beispielsweise Thumbtack. Diese Klassifikation mit ca. 500 digitalen Services auf Blattebene erhebt keinen Anspruch auf Vollständigkeit, sondern repräsentiert eine Stichprobe von etwa $0,001 \%$, wenn man von 5 Mio. digitalen Services jeder Art ausgeht. Sie soll lediglich die heute im Markt verfügbare Funktionalität anhand von Beispielen sichtbar machen. Sie ist auf IL.iwi.unisg.ch/AppMap (Oesterle 2014) abrufbar.

Dass digitale Dienste nicht nur verfügbar, sondern tatsächlich im Einsatz sind, zeigt eine Studie, wonach $80 \%$ der Schweizer Bevölkerung ab 14 Jahren das Internet mehrmals pro Woche nutzen. E-Mail, Presse, Information und Administration

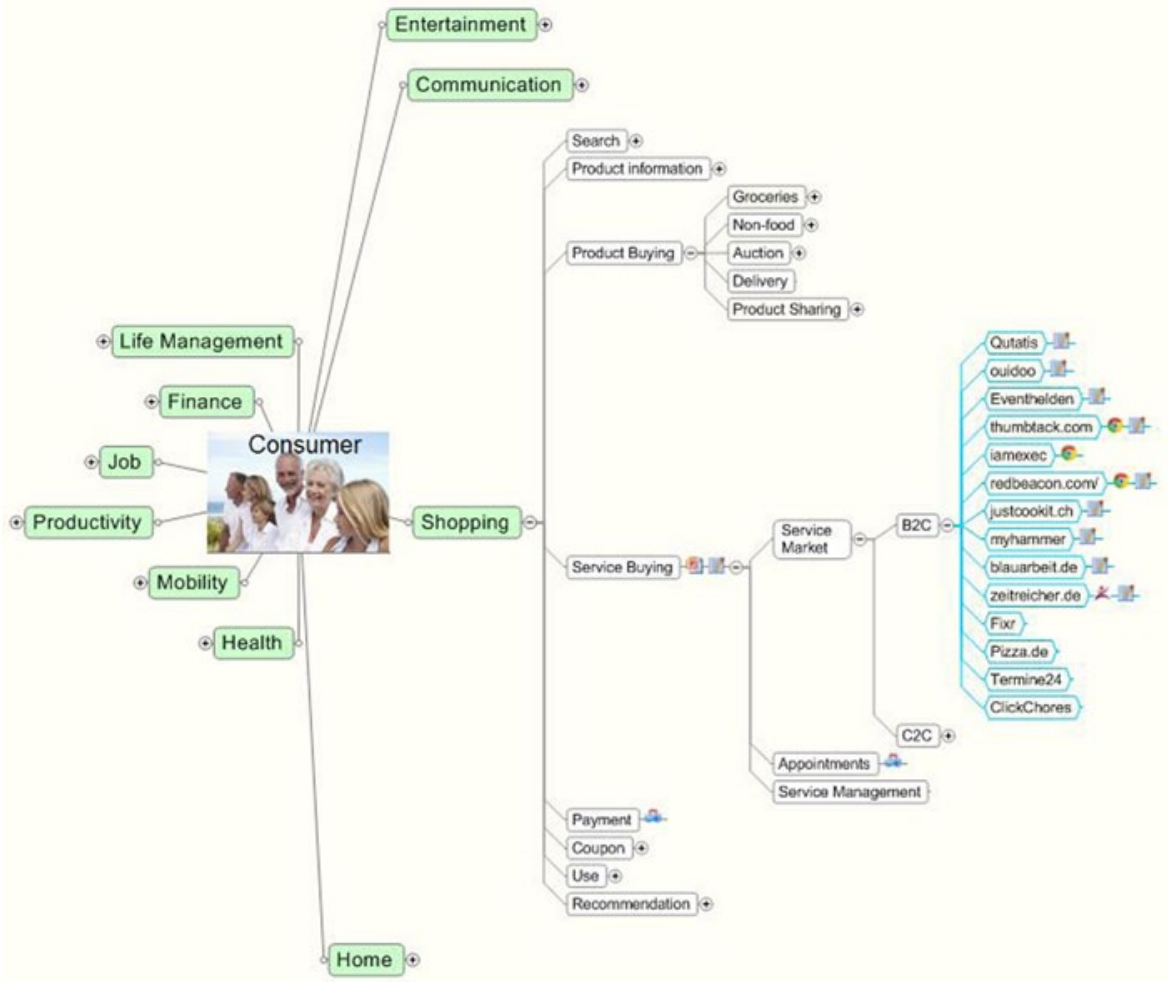

Abb. 1 Lebensbereiche und digitale Dienste. (Quelle: IL.iwi.unisg.ch/AppMap) 
Online-Aktivitäten für private Zwecke in den letzen drei Monaten, 2010

In \% der Internetnutzer (Internet in den letzten 3 Monaten genutzt)

E-Mails senden und empfangen

Nachrichten im Internet lesen, Zeitungen, Magazin usw.

Tätigkeiten im Zusammenhang mit der öffentlichen Verwaltung $\left(^{*}\right)$

Irgendetwas kaufen oder bestellen

Informationen suchen, die die Gesundheit betreffen

Dienstleistungen im Bereich

Reisen und Unterkunft nutzen

E-Banking, über Bank oder

Post- Konto Zahlungen machen

Filme und Videos herunterladen oder schauen

Musik herunterladen oder hören

Übers Internet Radio hören oder Fernseh schauen

Sein eigenes Profil auf einem sozialen Netzwerk haben

Sich informieren in Bezug auf politische Themen

Nachrichten senden via Chat, Forum, Newsgroup

Über Internet telefonieren oder Videokonferenzen

Computerspiele online spielen oder herunterladen

Eine Meinung äussern in Bezug auf politische Themen

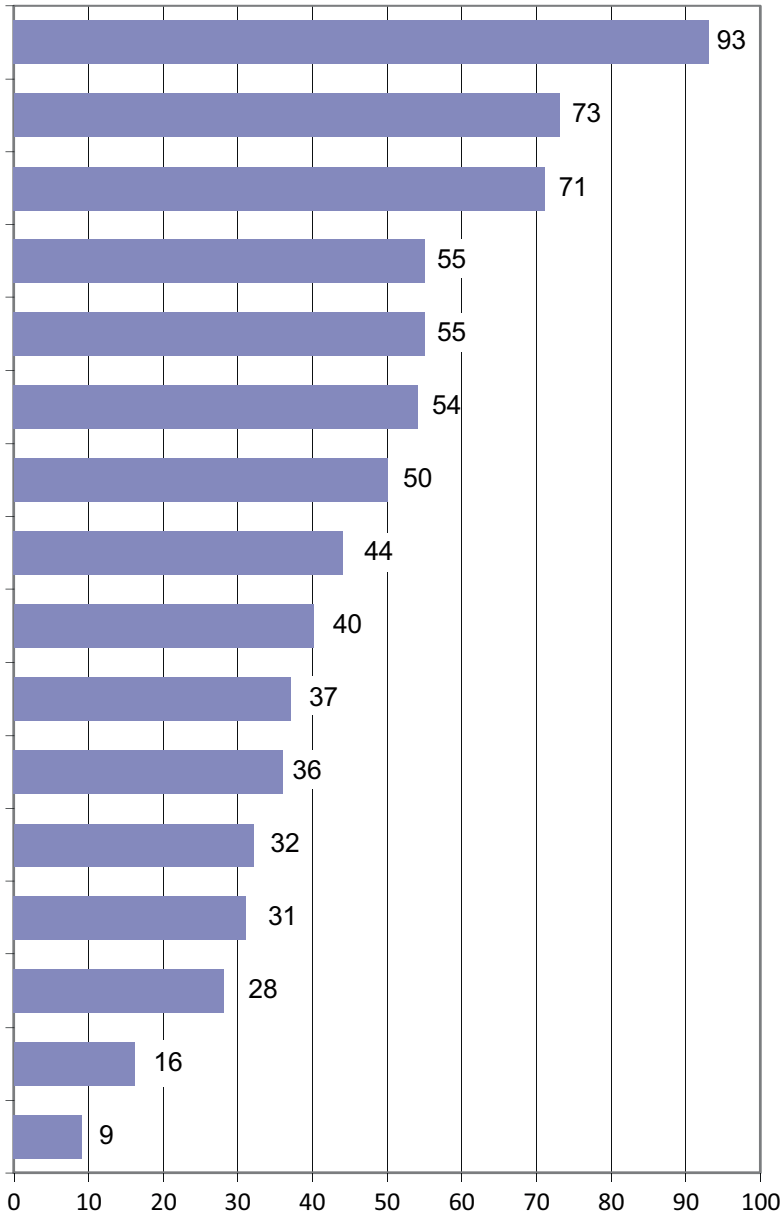

${ }^{*}$ ) In den letzten 12 Monaten

Abb. 2 Nutzung digitaler Services. (Quelle: Froidevaux (2012))

(öffentliche Verwaltung, Reisebuchung, Banking) dominieren die Nutzung (Abb. 2) (Froidevaux 2012).

\section{Geschäftsmodelle um den digitalisierten Konsumenten}

Brynjolfsson und McAfee (2014) zeichnen in ihrem Werk „The Second Machine Age: Work, Progress, and Prosperity in a Time of Brilliant Technologies" das Bild einer digitalisierten Wirtschaft mit grundlegend anderen Gesetzmäßigkeiten als wir sie aus der herkömmlichen Industriegesellschaft kennen. Geschäftsmodelle für das Kundensegment „Digitalisierte Konsumenten“ haben demnach wahrhaft giganti- 
sches Geschäftspotential. Nimmt man die Marktkapitalisierung von Unternehmen als Indikator für das Gewinnpotential der nächsten zehn bis 15 Jahre, so ragen Apple, Google, Amazon, Samsung, Tencent und ähnliche Unternehmen sowohl bezüglich ihrer Bewertung als auch bezüglich des Zuwachses der letzten Jahre weit aus dem Rest der Wirtschaft heraus. Einzelne Unternehmen können allerdings, wenn sie das Innovationstempo nicht halten oder sich strategisch falsch ausrichten, auch schnell massiv an Wert verlieren, wie dies die Mobiltelefonsparte von Nokia und RIM oder die Internetservices von AOL gezeigt haben.

Ähnliches gilt für Unternehmen mit klassischen Produkten. Versandhandel, Reisebüros, Medien (von Musik bis Tageszeitung), Verzeichnisdienste, Finanzdienstleister u. a. m. überleben oder wachsen, wenn sie ihr Produkt- bzw. Dienstleistungsportfolio rechtzeitig auf den digitalen Konsumenten ausrichten, scheiden sonst aber, wie viele Beispiele zeigen, aus dem Markt aus.

Gemäß einer Studie im Auftrag der EU (Mulligan und Card 2014) erwirtschafteten in der EU 1 Mio. App-Entwickler zus. mit 0,8 Mio. Marketing- und Administrationsmitarbeitern in 2013 einen Umsatz von 17,5 Mrd. EUR und erwarten in 2018 mit 2,7 Mio. Entwicklern und 2,1 Mio. Marketing- und Administrationsmitarbeitern einen Umsatz von 63 Mrd. EUR in der EU. Diese Erfolgsmeldung der Politik ist allerdings zu relativieren: Wenn mit den erwähnten Arbeitsplätzen Ganzzeitstellen gemeint sind, ergibt das einen Jahresumsatz von knapp 10.000 EUR pro Kopf. Außerdem erwartet man allein von Apple im gleichen Zeitraum ein Umsatzwachstum von 130 Mrd. EUR in 2013 auf 210 Mrd. EUR in 2018. Trotzdem gibt diese Studie einen Hinweis darauf, dass selbst in Europa die Wirtschaft zunehmend auf die Digitalisierung der Konsumenten setzt.

"The rapidly mutating mobile app market is an overclocked laboratory of capitalism“ (Kuittinen 2012). Die Gewinnchancen rund um die Digitalisierung der Konsumenten motivieren weltweit Millionen von Softwareentwicklern. In den Medien gefeierte Start-ups werden zu den Vorbildern einer ganzen Generation: WhatsApp erzielte innerhalb von knapp fünf Jahren nach der Gründung einen Verkaufspreis von 14 Mrd. EUR (davon Gründer Jan Koum 6.5 Mrd. EUR) (Schmid 2014), Palmer Luckey (mit 21 Jahren) knapp drei Jahre nach der Gründung von Oculus 800 Mio. EUR (Kyle und Tweedie 2014) und zuletzt Twitch.tv, das drei Jahre nach der Ausgründung aus Justin.tv für einen Preis von 730 Mio. EUR von Amazon gekauft wurde (Amadeo 2014).

Im Mittelpunkt der Geschäftsmodelle für digitale Konsumentenservices steht der Konsument, nicht der Anbieter der Produkte und Services. Diese scheinbare Trivialität bildet den Kern des Wettbewerbs zwischen Unternehmen wie Google, Apple und Microsoft (Arthur 2014). Der digitale Service muss Bedürfnisse und Gewohnheiten des Konsumenten treffen (Hrauda et al. 2002), sei es die Selbstdarstellung im Freundeskreis, sei es die Bequemlichkeit einer digitalen Navigation oder sei es einen Informationsbedarf. Noch viel wichtiger als im Geschäft mit Unternehmenskunden ist dabei die sog. User Experience, also das emotionale Erlebnis, vom Design über die Marke bis zur damit verbundenen Community.

Wenn der Nutzen für die Konsumenten gegeben ist, entscheidet im Sinne des Netzwerkgesetzes (Briscoe und Reed 2006) die Anzahl der Teilnehmer des Netzwer- 
kes über den Erfolg, was für die meisten Unternehmensbewertungen entscheidender als der erzielte Umsatz oder der Gewinn ist.

Volumen und damit Marktanteile bestimmen darüber, welche Ökosysteme überleben. Derzeit prägen Apple, Google, Amazon und Microsoft die technischen Ökosysteme für Konsumentenservices. Tim Cook, CEO von Apple, sieht das Apple Betriebssystem iOS in allen Bereichen des Lebens, vom Laptop oder Tablet zum Auto, zum Arbeitsplatz, zum Heim und zum Gym (Hughes 2014). Samsung und Sony versuchen, Alternativen wenigstens für Teilbereiche der Konsumentenbedürfnisse aufzubauen.

\section{Paradigmenwechsel 1: Vom Unternehmen zum Konsumenten}

Die Nutzung der Informationstechnik zur Steigerung von Effektivität und Effizienz von Unternehmen war und ist das Paradigma des Business Engineering. Produkte, Dienstleistungen, Wissen über das Kundensegment, Image im Markt, Ökosystem, Geschäftsprozesse und Organisationsstruktur sowie Preis und Kosten sind wichtige Hebel zur Steigerung des Unternehmenswertes (Kagermann et al. 2010).

Kundenzentrierung heißt für Unternehmen, die Erwartungen der Kunden an die Produkte und Dienstleistungen des Unternehmens zu kennen, die gemeinsame Historie mit dem Kunden vor Augen zu haben, Produkte und Dienstleistungen möglichst individualisiert zum Kunden zu bringen und ihn schließlich bei der Benutzung bis hin zur Entsorgung der Produkte zu begleiten, nach Möglichkeit also den gesamten Kundenprozess zu unterstützen (Tietz 2007).

Die deutschen Forschungs- und Entwicklungsinitiativen „Industrie 4.0“ und „Smart Service Welt“ (Kagermann 2014) folgen dem Paradigma der Unternehmenssicht. Sie nutzen die neuen Möglichkeiten des Internets of Things (Fleisch et al. 2014), um die Effizienz in der Produktion und Logistik zu steigern. Sie machen aber noch einen entscheidenden, weiteren Schritt, sie binden in ,Industrie 4.0“ die Unternehmenskunden (B2B) in die Leistungserbringung ausgehend von deren Prozessen (Kagermann et al. 2014) ein und schaffen in der Initiative „Smart Service Welt“ auch Grundlagen für Konsumentenservices.

Im Falle von B2C ist der Kunde der Konsument. Publikationen und Anleitungen zur Strategie- oder Geschäftsmodellentwicklung (Osterwalder und Pigneur 2011; Gassmann et al. 2013) blicken vom Unternehmen auf die Konsumenten. Da es um die Wertsteigerung von Unternehmen geht, ist dieses Vorgehen nachvollziehbar, es hat aber zur Folge, dass die Lösungen die Sicht einzelner Unternehmen, nicht die der Konsumenten reflektieren. Die Konsumenten müssen ihre Lebensbereiche selbst organisieren und stehen dabei vor einer unglaublichen und rasch wachsenden Vielfalt von inkompatiblen Services, die sie weder überblicken noch miteinander verbinden können. Es entstehen Geschäftsmodelle, aber keine Konsumentenmodelle. Die Aufgabe einer großen Zahl von Marktforschungseinrichtungen, aber auch der wissenschaftlichen Konsumentenforschung ist es, Konsumbedürfnisse, Zahlungsbereitschaft, Wettbewerbssituation usw. aus der Sicht des Markterfolges zu beleuchten, kaum aber dem Konsumenten zu helfen, einzelne Lebensbereiche mit digitalen Services zu organisieren. 
Apple hat wie kaum ein anderes Unternehmen vorgeführt, wie nicht der Anbieter, sondern der Konsument der Ausgangspunkt sein kann. So haben bereits der iPod und der iTunes Store den Konsumenten viele Aufgaben im Lebensbereich „Musik konsumieren" abgenommen. Die erwähnte Vision von Tim Cook von iOS in allen Lebensbereichen setzt die Strategie mit dem Konsumenten im Mittelpunkt fort. Das „Modell des Konsumenten" reicht von der Emotion (Marke, Vergleichsgruppe, Design) über die Funktionalität, die Geschäftsbedingungen (z. B. bezüglich Musikrechten) bis zum Betriebssystem. Es nimmt dem Menschen so viel Komplexität wie möglich ab. Ähnliche Ansätze sind bei Reiseservices wie Tripadvisor, ja sogar bei E-Banking mit immer mehr Services für die private Finanzadministration zu beobachten.

Eric Schmidt, damals noch CEO und heute Chairman von Google, formulierte 2011 Life Management der Konsumenten als Vision von Google. In seinem Buch zusammen mit Jared Cohen (Schmidt und Cohen 2013, S. 26 ff.) detailliert er die dafür notwendigen digitalen Services. Konsumentenportale wie Google wollen den Menschen bei der Auswahl des Restaurants, bei der Gesundheitsvorsorge und bei der finanziellen Altersvorsorge beraten. Sie fungieren dabei als Matchmaker, analysieren anhand von Big Data die Bedürfnisse und Präferenzen der Konsumenten und stellen diesen die passenden Angebote aufgrund ihres Zugangs zur Anbieterseite gegenüber. Sie entwickeln und vermarkten digitale Assistenten für ganze Lebensbereiche gemäß dem Motto „Focus on the user and all else will follow.“ (Google 2014).

Vor etwa 15 Jahren verfolgten Telekommunikationsunternehmen die Vision, dass sie das Portal der Konsumenten zu den Diensten des Internets werden. Sie investierten in digitale Services, haben aber ihr Ziel im Gegensatz zu den Geräte-, Software- und Serviceanbietern nicht erreicht. Google, Apple, Microsoft mit ihren Betriebssystemen, Facebook, Amazon und eBay sowie Booking.com mit starken Konsumentenservices haben diese Position eingenommen. Von China aus stoßen Alibaba und Tencent (vor allem über WeChat) in diesen Markt. Sie setzen auf den Komfort für den Konsumenten, nur ein Portal bedienen zu müssen. Diese Portale sind nicht nur der Zugang zu rein digitalen Services (von der Suche bis zum Chat), sondern auch zu physischen Produkten und physischen Dienstleistungen. Wenn die Konsumenten immer mehr über diese Portale einkaufen und die Hersteller die Angebote zunehmend über den Online-Kanal individualisieren, gewinnen diese Portale eine gewaltige Marktmacht, die im Einzelhandel und im Reisegeschäft bereits deutliche Spuren hinterlassen hat. Sie wird auch für die produzierende Industrie relevant, wenn Portale, wie die genannten, das Customer Relationship Management im B2CGeschäft der Konsumgüterhersteller übernehmen.

Ohne dies statistisch belegen zu wollen, hat die Digitalisierung der Konsumentenwelt doch zu einer vielerorts beobachtbaren Konsumentenzentrierung der Geschäftsmodelle geführt. Wenn man diese Entwicklung mit dem Stand vor zehn bis 15 Jahren vergleicht, darf man wahrscheinlich von einem Paradigmenwechsel im Business Engineering sprechen, weg von einer Sicht aus dem anbietenden Unternehmen auf viele Konsumenten hin zu einer Sicht des Konsumenten auf die Angebote an Produkten und Dienstleistungen vieler Unternehmen (s. Abb. 3). Für die meisten Unternehmen ist es bis heute allerdings mehr eine Herausforderung als eine Fähigkeit.

Wenn schon nicht die Wissenschaft, allen voran die Wirtschaftsinformatik, so hat doch die Wirtschaft mit einer Vielzahl von digitalen Assistenten für alle Lebensberei- 

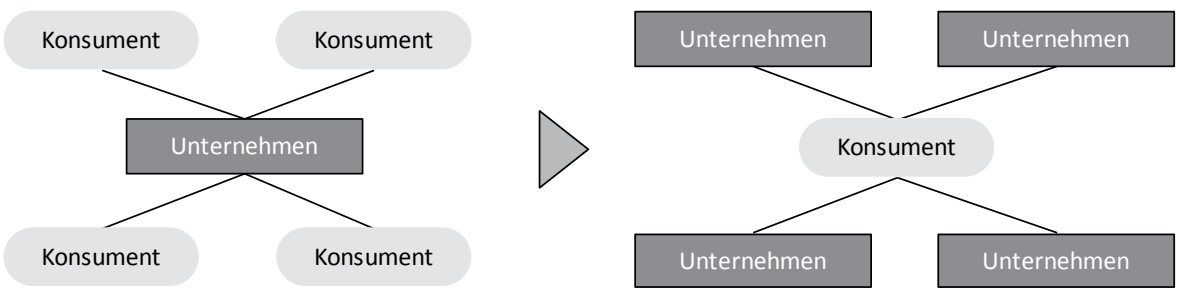

Abb. 3 Von der Unternehmenszentrierung zur Konsumentenzentrierung

che die Prioritäten geändert: Ausgangspunkt der Innovation wird die Konsumentenlösung, aus der dann alle unternehmensinternen Veränderungen abgeleitet werden.

\section{Paradigmenwechsel 2: Vom Konsumenten zum Menschen}

Das Ziel des Business Engineering ist eine nachhaltige Wertsteigerung der Unternehmen, also des Shareholder Value, auch wenn diese rationale Unternehmenszentrierung durch die Motivation und Begrenzungen der handelnden Mitarbeiter überlagert ist. Vereinzelt wird die Sicht des Shareholder Value wenigstens ansatzweise durch das Stakeholder Value, also den Blick auf die Anspruchsgruppen, erweitert.

Der Gewinn entscheidet über die Lebensfähigkeit von Unternehmen, die Wirtschaftlichkeit über die Verwirklichung von Projekten, der Preis über den Verkauf von Gütern und Diensten und die Produktivität letztlich über den Lohn des Mitarbeiters. Diese Steuerung der Wirtschaft über das Geld hat den Menschen in vielen Staaten einen hohen Lebensstandard im Sinne einer Versorgung mit Gütern und Dienstleistungen gebracht, die weit über die Befriedigung der Grundbedürfnisse hinaus geht.

Wirtschaft und Unternehmen sind jedoch kein Selbstzweck, sondern Konstrukte, die Menschen zur effizienteren, arbeitsteiligen Befriedigung ihrer Bedürfnisse und zum Austausch von Marktleistungen entwickelt haben. Die Abdeckung der Grundbedürfnisse in den hoch entwickelten Gesellschaften und die rasch wachsenden Möglichkeiten der Technik zur Schaffung einer schier unendlichen Zahl neuer Produkte und Dienstleistungen konfrontiert uns zunehmend mit der Frage, ob die Angebote den Menschen dienen oder die Menschen den anbietenden Unternehmen. Die einleitende Frage nach dem Nutzen für den Menschen geht weit über den Geschäftsnutzen hinaus.

Die Konsumenten sind gewöhnlich frei in ihrer Entscheidung für Produkte und Dienstleistungen. Es ist zu vermuten, dass sie jene Angebote kaufen, von denen sie den größten Nutzen erwarten, und die Anbieter sich Mühe geben, diese Bedarfe zu finden und zu befriedigen. Wenn diese Überlegung zutrifft, führt die Kreativität der Unternehmer, die jeden Winkel menschlicher Bedürfnisse auslotet, in eine immer bessere Welt der Konsumenten.

Es sei erlaubt, diese auf den ersten Blick schlüssige Logik infrage zu stellen:

- Entscheiden Menschen beim Kauf im Sinne eines momentanen Kaufimpulses oder einer dauerhaften Lebensqualität? Bedeutet der Konsum von Tabak, Alko- 
hol, Energy-Drinks und Süßwaren Glück oder überwiegen die negativen Folgen wie Lungenkrebs, Adipositas und andere Gesundheitsschäden?

- Kann der Konsument den Wert eines Produktes oder eines Services für seine Lebenssituation überhaupt ausreichend beurteilen? Etwa den Wert eines strukturierten Bankproduktes für die Altersvorsorge, eines Telekommunikationsvertrages oder eines 300-PS-Motors im Auto.

- Bringen die von allen Medien und insbes. der Werbung vermittelten Werte tatsächlich Lebensqualität für die Menschen? Erzeugt der statusgetriebene Kauf von Edelmarken tatsächlich dauerhaftes Glück oder führt der Wettbewerb der Statussymbole in die Verschuldung?

- Dominieren Konsumgüter unser Leben? Verdrängen die Angebote der Unternehmen wirtschaftlich nicht interessante Leistungen wie einen Spaziergang, ein Gespräch mit Freunden oder das Züchten eines Baumes aus einem Kirschkern.

- Führen die digitalen Services zu einer menschenfeindlichen Beschleunigung mit gesundheitlichen Schäden? Ist der drastische Anstieg der Frühberentungen (INQA 2013) eine Folge davon?

- Gefährdet die bewusste und die verborgene Weitergabe persönlicher Daten, also die Preisgabe der Privatheit, die Freiheit der Menschen?

Bereits diese Fragen lassen erkennen, dass wir uns, insbes. als Wirtschaftsinformatiker, hier auf sehr unsicherem Boden bewegen. Lebensqualität ist wohl das, was verschiedene Disziplinen als Glück bezeichnen. Einige Philosophen wie Epikur formulierten Glück als Abwesenheit von Schmerz. Religionen schufen Gebote für schmerzärmeres Zusammenleben und die Hoffnung auf Glück im Jenseits. Michael Argyle begründete eine Psychologie des Glücks (Argyle 2002), Gerhard Schulze sieht das Glück aus einer soziologischen Perspektive (Schulze 2000), Bjoern Grinde aus einer evolutionspsychologischen (Grinde 2002). Psychologen wie Maslow (1943) oder in jüngerer Zeit Reiss (2004) fokussieren auf die Bedürfnisse der Menschen. Schließlich sind auch die neurophysiologischen Glücksforschungen wie etwa von Esch (2013) zu erwähnen, die Glück als neurologisches Ereignis begreifen. FritzSchubert (Fritz-Schubert 2008) oder Gimpel et al. (2014) setzen Erkenntnisse der Glücksforschung in konkrete Anleitungen zur Förderung des Glücks um.

Trotz dieser vielfältigen Ansätze der Glücksforschung haben wir wenig klare Hinweise darauf, welche digitalen Services Menschen glücklich machen oder anders formuliert, zur Lebensqualität beitragen, also dauerhaften Nutzen produzieren. Will man die Erkenntnisse der Glücksforschung für die Digitalisierung zusammenfassen, so könnte man formulieren:

- Digitale Services sollen helfen, Schmerz zu vermeiden. Beispiele sind die Reduktion der Anstrengung, etwa bei der Suche nach Information, die Vermeidung von Umwegen bei der Navigation oder die Erhöhung der Sicherheit durch eine Alarmanlage.

- Digitale Services sollen helfen, die Grundbedürfnisse des Menschen (Essen, Trinken, Sex sowie Sicherheit und Liebe) zu befriedigen. Diese Aussage überlappt sich weitgehend mit der Vermeidung von Schmerz. 
- Wenn Schmerz vermieden und die Grundbedürfnisse befriedigt sind, dann können digitale Services dazu beitragen, Bedürfnisse wie Macht, Status, Ehre, Aussehen, Wissen und Kontakte, also den Menschen als soziales Wesen zu verwirklichen. Diese Bedürfnisse entstammen einem evolutionären Selektionsmechanismus, der die Weitergabe der Gene der Stärksten fördert (Grinde 2002).

Die Voraussetzungen für eine weitgehende Befriedigung der ersten beiden Punkte (Glück 1 nach Schulze) sind heute in den hoch entwickelten Gesellschaften gegeben. Digitale Dienste, herausragende Beispiele sind Facebook, WeChat, Tinder oder Youtube, versprechen daher immer mehr die Erfüllung der Selektionsbedürfnisse, also eine starke Positionierung in der Gesellschaft (Glück 2 nach Schulze). Das Problem daran ist, dass der Fortschritt des einen Menschen gleichzeitig der Rückschritt eines anderen ist, da in einer Rangordnung Aufstieg des Einen Abstieg des Anderen bedeutet.

Diese sehr rudimentären Überlegungen zur Ausrichtung der Digitalisierung auf den dauerhaften Nutzen des Menschen, auf die Lebensqualität, sind nicht als Antworten, sondern lediglich als Anregungen zur Beschäftigung mit diesem Thema zu verstehen. Wenn man der Überlegung zustimmt, dass der finanzielle Nutzen für ein Unternehmen nicht automatisch Lebensqualität der Konsumenten bedeutet, dann muss die Gesellschaft wohl einen Paradigmenwechsel ins Auge fassen: von der Steigerung des Unternehmenswertes zur Steigerung der Lebensqualität, also von der Geschäftssicht zur Menschensicht.

\section{Life Engineering}

Die Konsequenz aus diesem Paradigmenwechsel wäre eine Disziplin des Life Engineering, nicht anstatt, sondern zusätzlich zum Business Engineering, das weiterhin für Effizienz in der Befriedigung der Bedürfnisse sorgen muss. Life Engineering beschäftigt sich mit der Lebensqualität, den Bedürfnissen und den Möglichkeiten zu deren Befriedigung sowie dem Beitrag der digitalen Services.

Technologieanbieter wie Apple, Google, Samsung oder Tencent integrieren immer mehr Services in ihr Angebot und wollen damit den Konsumenten in allen Lebensbereichen unterstützen. Der Navigationsservice beispielsweise nutzt nicht nur Lokalisierung und Kartenmaterial, sondern auch Verkehrsnachrichten, Verkehrsflussinformationen von anderen Fahrzeugen, den Kalender und das Adressbuch des Konsumenten sowie seine Navigationshistorie (,zuletzt benutzte Adresse“).

Die Entwicklung von Life Management Lösungen durch Google und andere Technologie- und Serviceanbieter kann und wird die Wirtschaftlichkeit von konsumentenzentrierten digitalen Services voran bringen und unternehmerisch interessante Services präsentieren. Digitale Services werden aber die Lebensqualität der Konsumenten nur dann verbessern, wenn Unternehmenserfolg und Lebensqualität zusammen fallen, was für viele Services durchaus der Fall ist, aber nicht als grundsätzlich gegeben gelten kann.

Das Ziel eines Life Engineering ist es, durch digitale Dienste die Lebensqualität der Menschen nachhaltig zu verbessern. Wessen Aufgabe ist aber Life Engineering? 
Wer hat die Voraussetzungen, also Fähigkeiten und Ressourcen, um sich mit den Grundlagen der Lebensqualität auseinander zu setzen und die Erkenntnisse in die digitalen Dienste einzubringen?

Der Mensch, also das Individuum, ist überfordert. Er bekommt weder einfach anwendbare Erkenntnisse aus der Glücksforschung noch hat er die Möglichkeiten, aus den millionenfachen Angeboten fundiert auszuwählen, Services zu kombinieren, die Entwicklung der digitalen Dienste zu beeinflussen oder diese für sich anzupassen (Konsumentenmodell). Die Wirtschaftsunternehmen folgen dem Paradigma der Wirtschaftlichkeit. Der Staat und die Politik beschäftigen sich durchaus mit einzelnen Themen der Lebensqualität wie etwa dem Verbot von Tabakwerbung, den Inhaltsstoffen von Lebensmitteln und dem Persönlichkeitsschutz oder der Abwehr von Monopolen, lassen aber noch kaum Vorstöße zum Umgang mit digitalen Diensten erkennen, wie dies Frank Schirrmacher monierte, der im Zusammenhang mit Google von einer neuen digitalen Planwirtschaft sprach (Schirrmacher 2013), die Dave Eggers in Form des Romanes „The Circle“ ein paar Jahre in die Zukunft weiter entwickelt hat.

Zivilgesellschaftliche Organisationen könnten die Führung im Life Engineering übernehmen. Viele von ihnen suchen und schaffen Werte und Lebenssinn über den Konsum hinaus. Tausende von Freiwilligenorganisationen, Tauschbörsen, Zeitbörsen, Stiftungen und nicht zuletzt politische und religiöse Organisationen sind Belege dafür (Gesinke 2011). Es fehlen ihnen zwar die Ressourcen und die Kultur zum Aufbau von Alternativen für Konsumentenportale, doch können sie möglicherweise Anstöße geben, das Konsumentenverhalten und damit das Angebot der Unternehmen beeinflussen.

In den wissenschaftlichen Journalen und Konferenzen zu Informationssystemen, digitalen Services und Konsumenten findet man wenig zu den Fragen des Life Engineering, wenn man von den Themen Privatheit und Konsumentenschutz absieht.

Life Engineering muss Antworten auf Fragen wie die folgenden liefern:

- Wie kann ein Individuum seine Grundbedürfnisse, insbes. die Vermeidung von Schmerz, über sein gesamtes Leben befriedigen? Themen darin sind Ausbildung und Berufswahl, Gesundheitsvorsorge, finanzielle Sicherheit und schließlich Hilfe und Pflege im Alter, Vorsorgevollmacht, Patientenverfügung oder Exit-Vereinbarung. Kann ein digitaler Assistent wie swisshelp66 dabei helfen?

- Können digitale Assistenten das Wohlbefinden im Sinne der Mind-body Medicine (Gimpel et al. 2014) fördern?

- Wie kann das Individuum sein Glück über die Grundbedürfnisse hinaus beeinflussen? Können digitale Dienste wie etwa soziale Netzwerke beispielsweise das Selbstwertgefühl nicht nur einzelner, sondern aller Teilnehmer steigern?

- Wenn Unternehmen nur wirtschaftlich interessante Services anbieten, sollen dann Vereine, Religionsgemeinschaften und andere zivilgesellschaftliche Organisationen verstärkt andere Leistungen wie beispielsweise einen Spaziergang bieten?

- Kann ein persönlicher Administrationsassistent den Menschen die Last der Verwaltungsaktivitäten („Konsumenten-ERP“) abnehmen oder mindestens die Komplexität reduzieren? 
- Wie kann dem Menschen die Suche, die Konfiguration, die Integration, die Privatheit, das Update, die Synchronisation, die Datensicherung usw. dieser Dienste abgenommen werden?

- Wer bestimmt, was für den Einzelnen gut ist? Das Individuum, der Staat, die Religion,...?

- Wie kann ein digitaler Assistent die individuellen Ziele und Prioritäten erfassen?

- Welche existierenden digitalen Services passen zu einem bestimmten Profil eines Konsumenten?

- Wie kann den Gefahren der Digitalisierung etwa in Form des Verlustes an Privatheit, des Mobbings auf sozialen Netzwerken oder der Forcierung schädlichen Konsums begegnet werden?

- Brauchen die Menschen eine Ausbildung in Lebensqualität und als Teil davon eine Anleitung zur Nutzung und Organisation ihrer digitalen Umwelt?

Viele dieser Fragen sind nicht neu, doch die Digitalisierung bringt vollkommen neue Möglichkeiten, die eine Beschäftigung mit derartigen Fragen als dringend notwendig erscheinen lassen.

\section{Life Engineering am Beispiel des digitalen Dorfes}

Globalisierung, Mobilität, Reurbanisierung, Demographie, digitale Gemeinschaften und weitere Trends zerstören soziale Beziehungen auf dem Dorf oder im Quartier der Stadt. Die Folge sind Schmerzpunkte wie Einsamkeit, Unsicherheit und Unterstützungsbedarf bei Alltagsaufgaben, insbesondere bei älteren Menschen. An der Universität St. Gallen läuft seit sechs Jahren das Forschungsprojekt „Independent Living“. Sein Ziel ist es, für Senioren, aber auch für alleinerziehende Mütter, für vielbeschäftigte Doppelverdiener und ähnliche Zielgruppen einen Ersatz für Dorf- bzw. Quartiersstrukturen zu schaffen. Die Ansatzpunkte des Vorhabens sind:

- Erleichterung der Kommunikation in einem Quartier: Das bedeutet sowohl die globale Kommunikation wie z. B. von Großeltern mit ihren Enkeln irgendwo auf der Welt als auch die Verstärkung der Kommunikation mit Nachbarn.

- Gemeinschaft: Digitale Services verstärken lokale Interessengemeinschaften wie Vereine und schaffen zusätzliche räumlich nicht gebundene Gemeinschaften.

- Inhalte: Digitale Bürgerforen, Quartierszeitungen und Anschlagbretter liefern sehr lokale Neuigkeiten mit direkter Relevanz für die Quartiersbewohner.

- Koordination: Den angesprochenen Zielgruppen fehlt das klassische Dorf oder Quartier vor allem bei der Organisation von Alltagsaufgaben: das Finden eines zuverlässigen Babysitters, die vertrauenswürdige Betreuung von Wohnung, Pflanzen und Haustieren bei längerer Abwesenheit, die Vereinbarung eines Termins beim Arzt oder die zeitpunktgenaue Lieferung von Lebensmitteln. Zusätzlich zu all den professionellen Dienstleistern existieren an vielen Orten Zeitbörsen, Tauschbörsen, nachbarschaftlich organisierte Sicherheitssysteme und soziale Organisationen. Die Versorgung mit physischen Dienstleistungen ist vorhanden, erfordert aber einen hohen Organisationsaufwand, der teilweise an Öffnungszei- 
ten gebunden ist. Die Vertrauenswürdigkeit und die Bewältigung von Ausnahmesituationen in der Abwicklung der Dienstleistungen verlangen persönlichen Einsatz von Intermediären.

Viele Vertreter der Zielgruppen nutzen heute schon Facebook, Skype oder WhatsApp und E-Mail für die Dorffunktionen Kommunikation, Gemeinschaft, Informationen und Neuigkeiten. An einigen Orten verwenden sie digitale Nachbarschaftsservices wie NextDoor, i-Neighbors oder Netzwerk-Nachbarschaft. Auch für die Koordination von Dienstleistungen gibt es bereits einige Ansätze, von Dienstleistungsportalen wie MyHammer über Angie's List, redbeacon und Termine24 bis zu Thumbtack und Mila (s. Zweig Shopping und Home in IL.iwi.unisg.ch/AppMap). All diesen organisatorischen und technischen Lösungen ist gemein, dass sie noch nicht wirtschaftlich nachhaltig funktionieren. Eine Ursache ist das Verhalten der Konsumenten, die wie in der Anfangszeit des electronic Banking weiterhin die vertrauten Wege gehen. Ein weiterer Grund liegt möglicherweise darin, dass die Lösungen die Bedürfnisse der Konsumenten nicht treffen.

Das Projekt Independent Living ist von der Vision des digitalen Dorfes geleitet, in dem digitale Services alte Dorfstrukturen ersetzen oder bestehende verstärken sollen. Startpunkt ist das Management von Alltagsaufgaben über die Plattform Amiona. Konsumenten können auf Amiona Dienstleistungen von professionellen Dienstleistern oder Nachbarn inklusive Termin buchen, abwickeln und bewerten (zu Dienstleistungsplattformen s. Riemer und Vehring 2012).

In der baden-württembergischen Kleinstadt Weil der Stadt läuft seit 2012 der Pilotversuch „Lebensqualität Weil der Stadt“ auf einer ersten Version der Plattform. Aus ihr entstand eine verbesserte Lösung (s. Abb. 4), die nun zusätzlich in St. Gallen (Schweiz) in einem nächsten, größeren Pilotversuch eingesetzt wird. Das wichtigste Ziel der Pilotversuche ist der Beweis, dass die Abwicklung von Alltagsaufgaben über eine App bzw. eine Plattform den Konsumenten so viel Nutzen bringt, dass diese sie im täglichen Leben einsetzen. Die App muss also eine Dienstleistung einfacher als über Telefon abwickeln. Das zweite Ziel ist die Akzeptanz der Plattform durch die Dienstleister, doch scheint dies anhand des ersten Pilotversuchs bereits gesichert.

Amiona adressiert konkrete Schmerzpunkte der Adressaten. Sie reduziert die Komplexität und den Verwaltungsaufwand, ist $7 * 24$ verfügbar und gibt Sicherheit durch den Intermediär, durch Bewertungen seitens der Konsumenten und durch Terminerinnerungen.

Die Vision des digitalen Dorfes und der Prototyp der Dienstleistungsplattform folgen dem Paradigma der Lebensqualität. Nicht der Geschäftserfolg, sondern die Lebensqualität ist der Maßstab für die Entwicklung von Amiona. Die Vermittlung von kostenlosen Dienstleistungen von Privatpersonen oder von Tauschgeschäften usw. erzeugt keine Erlöse, bringt aber Lebensqualität für die Nutzer. Einerseits können Konsumenten damit grundlegende Bedürfnisse abdecken, andererseits geben private Anbieter von kostenlosen Dienstleistungen ihrem Leben mehr Sinn.

Die Digitalisierung kann die Lebensqualität nur dann dauerhaft verbessern, wenn sie wirtschaftlich tragfähig ist. Entweder erzeugen die Services, also beispielsweise die Vermittlung professioneller Dienstleistungen Erlösströme, oder die Kommunen 
Abb. 4 Mobile App Amiona

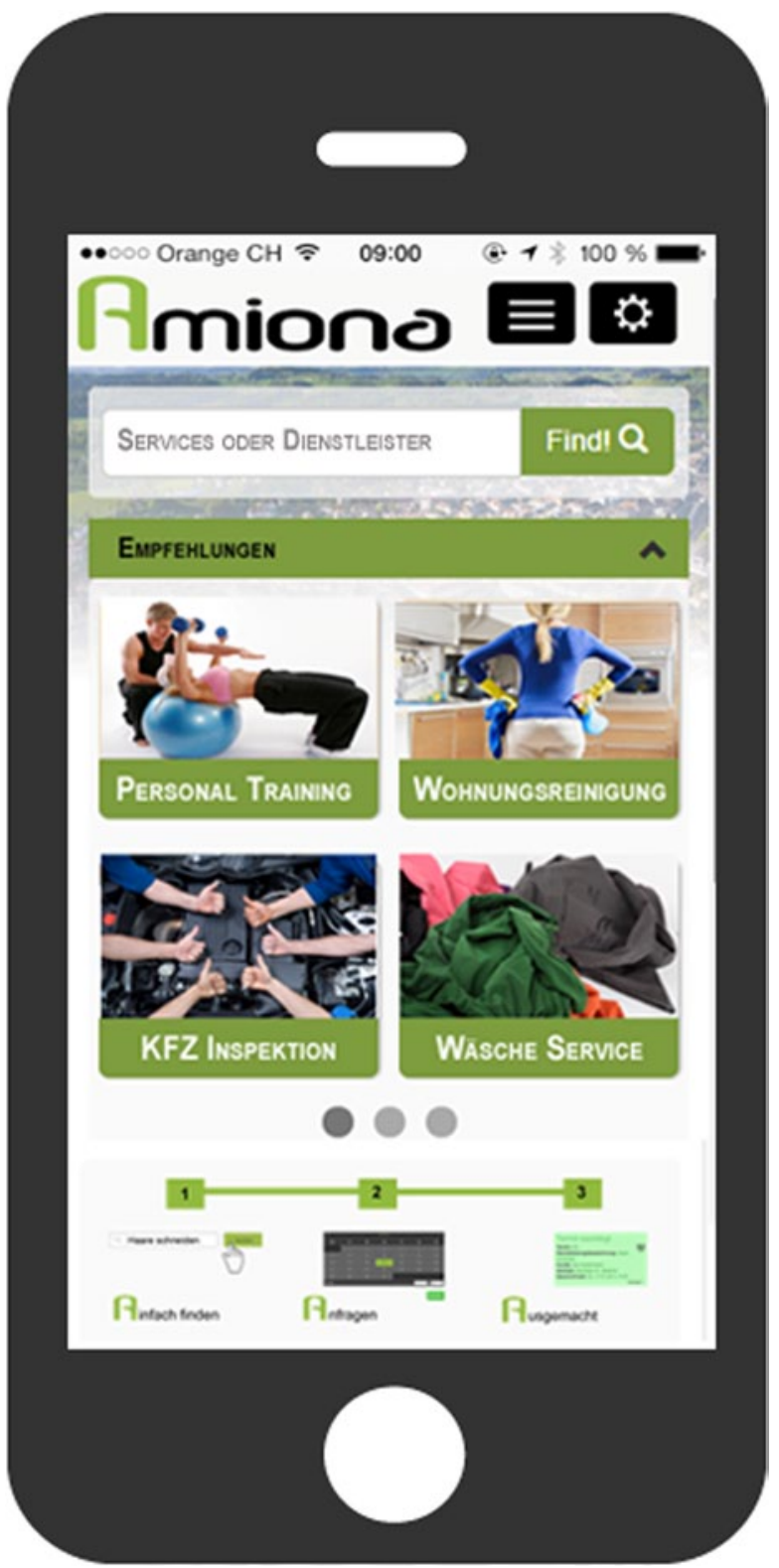

und andere Organisationen finanzieren den Betrieb derartiger Lösungen so wie den Betrieb des Straßennetzes.

Die Anbieter von Konsumentenportalen werden argumentieren, dass sie mit ihrem totalen Fokus auf den Konsumenten für höhere Lebensqualität sorgen. Wenn ausreichender Nutzen entsteht, wird dafür auch bezahlt. Im Beispiel des digitalen Dorfes heißt das aber, dass alles, was ausreichende Erlöse (z. B. über Werbung) generiert, realisiert wird, alles andere aber liegen bleibt. Es heißt eben, dass nicht die Leistung 
mit dem größten Wert für eine dauerhafte Lebensqualität, sondern die Leistung mit dem größten finanziellen Nutzen angeboten wird.

Das Beispiel des digitalen Dorfes und der Teillösung Dienstleistungsplattform veranschaulicht, dass Geschäftserfolg und Lebensqualität sich nicht widersprechen müssen, aber auch, dass sie nicht deckungsgleich sind.

Die derzeitige Realisierung über Amiona und bestehende Services (Facebook usw.) kann nur als ein erster, kleiner Schritt für die Erreichung von Lebensqualität bezeichnet werden. Wenn der Pilotversuch gelingt, zeigt dieses Beispiel, dass dem Paradigma Lebensqualität nachgelebt werden kann, wenn damit auch das Paradigma des Geschäftserfolgs erfüllt wird.

\section{Umsetzungs- und Forschungsagenda}

Die Unternehmen haben sich bereits in vielen Fällen von der internen Unternehmenssicht zu einer Kunden-, insbesondere Konsumentensicht bewegt und damit den Paradigmenwechsel vom Unternehmen zum Konsumenten vollzogen. Radikal vom Konsumenten aus denkende Unternehmen scheinen besonders erfolgreich zu sein. Für viele Unternehmen, insbes. im Bereich der Medien, der Finanzen oder der Gesundheit, bedeutet dieser Paradigmenwechsel noch ein gewaltiges Umdenken, weg vom effizienzgetriebenen Anbieten eines Produktes hin zum Verstehen des Konsumenten und seiner Prozesse.

Der zweite Paradigmenwechsel, vom Konsumenten zum Menschen, d.h. vom Unternehmenserfolg zur Lebensqualität, ist noch viel schwieriger. Zur Messung des Unternehmenserfolgs gibt es anerkannte und leicht erfassbare Messgrößen wie Umsatz und Gewinn, zur Messung der Lebensqualität gibt es vielfältige, aber zur Beurteilung von digitalen Services kaum verwendbare Indizes (Chassany et al. 2004).

Für den Paradigmenwechsel 1 gibt es klare Verantwortlichkeiten. Die Mitarbeiter der Unternehmen, von der Geschäftsleitung bis zum Mitarbeiter im Verkauf, müssen die Bedürfnisse der Konsumenten erkennen und in Angebote umsetzen. Für das Paradigma der Lebensqualität fühlen sich alle und damit gleichzeitig niemand zuständig.

Wenn die finanzielle Zielsetzung entfällt, entfallen die größten Ressourcen zur Entwicklung von Lösungen für die Lebensqualität. Allerdings, wenn eine Disziplin des Life Engineering praktisch verwendbare Antworten liefert, können Unternehmen sie im Business Engineering nutzen. In diesem Fall sorgt die finanzielle Steuerung für die Ressourcen zur Steigerung der Lebensqualität. Ein Beispiel könnte die individualisierte und digital gesteuerte Medikation bei Krankheiten sein. Vermeidung von Schmerz und Unternehmenserfolg fallen zusammen.

Schwieriger wird es, wenn die Lebensqualität langfristige Ziele verfolgen muss, die Konsumenten aber kurzfristig entscheiden. Kurzfristige Ernährungsentscheidungen folgen nur bedingt längerfristigem Wohlbefinden, wie Adipositas und Alkoholsucht belegen.

Ein weiterer Zielkonflikt besteht, wenn die Lebensqualität Leistungen beansprucht, aus denen kein Erlös erwirtschaftet werden kann. Das Gespräch mit einem Freund, der Spaziergang oder die stille Meditation sind Leistungen für die Lebens- 
qualität, zu denen die Digitalisierung erstens nicht viel beitragen kann und aus denen sie zweitens kaum Erlöse erwirtschaften kann.

Life Engineering steht damit zwar vor äußerst anspruchsvollen Aufgaben, kann aber wohl, wie das Beispiel des digitalen Dorfes zeigen soll, inkrementell die Lebensqualität steigern. Ob die Wirtschaftsinformatik dazu beitragen kann und will, ist im Moment nicht erkennbar. Nimmt man die Publikationen in ihren sog. TopJournalen und Konferenzen als Indikation, so ist kaum damit zu rechnen. Hatte sich die Wirtschaftsinformatik in den siebziger Jahren intensiv mit den EDV-Anwendungen in den Unternehmen beschäftigt, so vermisst man eine ähnliche Diskussion von Applikationen der heutigen IT, vor allem im Konsumentenbereich, fast vollständig. Während in den siebziger Jahren auch eine Auseinandersetzung mit den Zielen von EDV-Projekten aus Sicht der Unternehmen stattfand, liest man nichts über die Ziele von Menschen als Nutzer von digitalen Diensten, also über die Lebensqualität.

Im Gegensatz zu den siebziger Jahren kommen aus der Wirtschaftsinformatik wenige Lösungsvorschläge oder Prototypen, da sich unsere Disziplin, getrieben durch eine Incentivierung über Publikationen in Mainstream-Journalen, auf die statistische Ergründung von Zusammenhängen geringer Relevanz ausgerichtet hat. Der Entwurf von innovativen Konzepten und Prototypen für den Konsumenten spielt im derzeitigen Wertesystem der Wirtschaftswissenschaften kaum noch eine Rolle. Umfassende Lösungen, welche die unzähligen partiellen Ansätze beispielsweise von mobilen Apps im Sinne der Konsumenten verbinden, sind derzeit kaum zu beobachten. Dabei könnte die quantitativ-empirische Forschung wertvolle Unterstützung liefern, wenn sie den Datenfundus der digitalen Serviceanbieter nutzte. Die Auswertung dieser Daten wird den Unternehmen überlassen und nicht publiziert.

Es steht zu befürchten, dass die Wirtschaftsinformatik weder zum Paradigmenwechsel 1 im Business Engineering noch zum Paradigmenwechsel 2 im Life Engineering viel beitragen wird.

Das 50-jährige Bestehen der HMD, einer Zeitschrift für praxisrelevante Forschung, ist eine Gelegenheit grundsätzlich darüber nachzudenken, wohin uns die Digitalisierung der Welt des Konsumenten führen kann und was daraus für die Wirtschaft, vor allem aber für die Menschen folgt. Der vorliegende Beitrag ist eine Aufforderung zum Nachdenken und präsentiert weder ein konkretes Artefakt noch eine empirisch fundierte Theorie, sondern ist ein Opinion Article. Er ist so weit vom Mainstream der Wirtschaft und Wissenschaft entfernt, dass er wenig auf gesicherten und publizierten Erkenntnissen aufbauen kann. Es ist schade, dass derartige Arbeiten in der momentanen Wissenschaftskultur jungen Forschern nicht zugestanden werden, und zu hoffen, dass so gewagte Überlegungen wenigstens einem alten Forscher nachgesehen werden.

\section{Business und Life Engineering}

Die Digitalisierung der Konsumentenwelt bringt einen Paradigmenwechsel für Unternehmen: Erfolgreiche digitale Dienste entstehen aus Sicht der Lebensbereiche von Konsumenten; die Leistungserstellung im Unternehmen leitet sich daraus ab. Die Digitalisierung der Welt legt uns nahe, über einen zweiten Paradigmenwechsel 
nachzudenken, also nicht nur dem Paradigma der Konsumentenzentrierung aus Sicht des Geschäftserfolgs, sondern auch dem Paradigma der Lebensqualität nachzuleben. Die Antwort auf die Frage im Titel dieses Beitrages lautet: Business UND Life Engineering, also Lebensqualität und effiziente Leistungserstellung. Wenn Wissenschaft und Gesellschaft keine Antworten finden, wird die Wirtschaft Lösungen ausschließlich aus geschäftlicher Sicht produzieren.

Open Access Dieser Artikel unterliegt den Bedingungen der Creative Commons Attribution License. Dadurch sind die Nutzung, Verteilung, und Reproduktion erlaubt, sofern der/die Originalautor/en und die Quelle angegeben sind.

\section{Literatur}

Amadeo R (2014) Amazon officially buys Twitch. http://arstechnica.com/gaming/2014/08/amazon-notgoogle-reportedly-buying-twitch-for-1-billion/. Zugegriffen: 20. Sept. 2014

Argyle M (2002) The psychology of happiness. Routledge, New York

Arthur C (2014) Digital wars: Apple, Google, Microsoft and the battle for the Internet. Kogan Page, London

Briscoe B, Reed, DP (2006) Metcalfe's Law is Wrong. IEEE Spectrum (July):34-39

Brynjolfsson E, McAfee A (2014) The second machine age: work, progress, and prosperity in a time of Brilliant Technologies. W. W. Norton \& Company, New York

Chassany O, Dimenäs E, Dubois D, Wu A (2004) The Psychological General Well-Being Index (PGWBI) user manual. Lyon. http://178.23.156.107:8085/Instruments_files/USERS/pgwbi.pdf. Zugegriffen: 20. Sept. 2014

Esch T (2013) Die Neurobiologie des Glücks. Georg Thieme, Stuttgart

Fleisch E, Weinberger M, Wortmann F (2014) Geschäftsmodelle im Internet der Dinge. Arbeitsbericht. http://www.iot-lab.ch/wp-content/uploads/2014/09/GM-im-IOT_Bosch-Lab-White-Paper.pdf. Zugegriffen: 20. Sept. 2014

Fritz-Schubert E (2008) Schulfach Glück. Wie ein neues Fach die Schule verändert. Herder, Freiburg

Froidevaux Y (2012) Internet in den Schweizer Haushalten: Ergebnisse der Erhebung Omnibus IKT 2010. Bundesamt für Statistik (BFS), Neuchâtel

Gassmann O, Frankenberger K, Csik M (2013) Geschäftsmodelle entwickeln: 55 innovative Konzepte mit dem St. Galler Business Model Navigator. Hanser, München

Gesinke T (2011) Freiwilligensurvey. In: T Olk, B Hartnuß (Hrsg) Handbuch Bürgerschaftliches Engagement. Juventa, Weinheim und Basel, S 691-704

Gimpel C, Esch T, Von Scheidt C, Michalsen A, Jose G, Sonntag U, Stefano GB (2014) Changes and interactions of flourishing, mindfulness, sense of coherence, and quality of life in patients of a mind-body medicine outpatient clinic. Forschende Komplementärmedizin 21(3):154-162

Google (2014) Ten things we know to be true. http://www.google.com/intl/en/about/company/philosophy/. Zugegriffen: 20. Sept. 2014

Grinde B (2002) Darwinian happiness: evolution as a guide for living and understanding human behavior. Darwin Press, Princeton

Hrauda G, Jasch C, Kranzl S, Horvath F (2002) Homeservices aus der Fabrik der Zukunft. Wien. http:// www.fabrikderzukunft.at/nw_pdf/0305_homeservices.pdf. Zugegriffen: 20. Sept. 2014

Hughes N (2014) UBS praises Apple CEO Tim Cook for thinking „big,“ pushing for iOS to become ubiquitous. http://appleinsider.com/articles/14/07/30/ubs-praises-apple-ceo-tim-cook-for-thinkingbig-pushing-for-ios-to-become-ubiquitous. Zugegriffen: 11. Sept. 2014

INQA (2013) Psychische Erkrankungen gewinnen rasant an Bedeutung. http://psyga.info/presse/\#c330. Zugegriffen: 17. April 2014

Kagermann H (2014) Industrie 4.0 und Smart Services. In: W Brenner, T Hess (Hrsg.), Wirtschaftsinformatik in Wissenschaft und Praxis. Springer Gabler, Berlin, S 223-249

Kagermann H, Österle H, Jordan J (2010) IT-Driven business models. John Wiley \& Sons, Hoboken 
Kagermann H, Riemensperger F, Hoke D, Helbig J, Stocksmeier D, Wahlster W, Scheer AW, Schweer D (2014) Smart Service Welt. Recommendations for the strategic initiative web-based services for businesses. Berlin.

Kuittinen T (2012) Angry Birds Star Wars and the evolution of the app market. http://bgr.com/2012/10/08/ mobile-app-market-analysis-angry-birds-star-wars/. Zugegriffen: 11. Sept. 2014

Kyle R, Tweedie S (2014). How a 21-year-old college dropout built the virtual reality company Facebook just bought for $\$ 2$ billion. http://www.businessinsider.com/how-a-21-year-old-college-dropout-builtoculus-2014-3. Zugegriffen:10. Sept. 2014

Maslow AH (1943) A theory of human motivation. Psychological Review 50:370-396

Mulligan M, Card D (2014) Sizing the EU app economy. http://ec.europa.eu/digital-agenda/en/news/ sizing-eu-app-economy. Zugegriffen: 10. Sept. 2014

Oesterle H (2014) Consumer App Map. IL.iwi.unisg.ch/AppMap. Zugegriffen: 18. Sept. 2014

Osterwalder A, Pigneur Y (2011) Business Model Generation: ein Handbuch für Visionäre, Spielveränderer und Herausforderer. Campus, Frankfurt a. M.

Reiss S (2004) Multifaceted nature of intrinsic motivation: the theory of 16 basic desires. Review of General Psychology 8(3):179-193

Riemer K, Vehring N (2012) Virtual or vague? a literature review exposing conceptual differences in defining virtual organizations in IS research. Electronic Markets 22(4):267-282

Schirrmacher F (2013) Das ist Googles Wille. Die neue digitale Planwirtschaft. http://www.faz.net/ aktuell/feuilleton/debatten/digitales-denken/das-ist-googles-wille-die-neue-digitale-planwirtschaft-12162503.html. Zugegriffen: 26. April 2014

Schmid S (2014) Facebook lässt sich nicht lumpen. Neue Zürcher Zeitung 21.2.2014:29

Schmidt E, Cohen J (2013) The new digital age. Reshaping the future of people, nations and business. John Murray, New York

Schulze G (2000) Die Erlebnisgesellschaft: Kultursoziologie der Gegenwart, 8. Aufl. Campus, Frankfurt a. M.

Tietz D (2007) Der Einfluss des Internets auf Intermediäre im Tourismus. DUV, Wiesbaden 